\title{
A New Regular Matrix Defined By Fibonacci Numbers And Its Applications
}

\author{
Murat KARAKAȘ*
}

Bitlis Eren Üniversitesi, Fen Edebiyat Fakültesi, İstatistik Bölümü, 13000,Bitlis

\begin{abstract}
The main goal of this paper is to define a new infinite Toeplitz matrix and to examine some algebraic and topological properties of the sequence spaces $l_{p}, l_{\infty}, c$ and $c_{0}$ where $1 \leq p<\infty$ by means of this matrix.

Keywords: Regular matrix, Fibonacci numbers, Sequence space
\end{abstract}

\section{Fibonacci Sayıları Yardımıyla Tanımlanan Yeni Bir Regüler Matris ve Uygulamaları}

\begin{abstract}
Özet
$\mathrm{Bu}$ çalışmanın temel amacı, Fibonacci sayılarını kullanarak bir sonsuz Toeplitz matrisi tanımlamak ve bu matris yardımıyla $1 \leq p<\infty$ olmak üzere $l_{p}, l_{\infty}, c$ ve $c_{0}$ dizi uzaylarının bazı cebirsel ve topolojik özelliklerini incelemektir.
\end{abstract}

Anahtar Kelimeler: Regüler matris, Fibonacci sayıları, Dizi uzayı

\section{Introduction}

By $w$, we shall denote the space of all real valued sequences. Each linear subspace of $w$ is called a sequence space. Let $l_{\infty}, c, c_{0}$ and $l_{p}(1 \leq p<\infty)$ be the linear spaces of bounded, convergent, null sequences and p-absolutely convergent series, respectively.

Suppose $A=\left(a_{n k}\right)$ is an infinite matrix of real numbers $a_{n k}$, where $n, k \in I N$ and $x=\left(x_{k}\right) \in w . \quad$ We $\quad$ write $\quad A x=\left(A_{n}(x)\right) \quad$ if $\quad A_{n}(x)=\sum_{k} a_{n k} x_{k}$ converges for each $n \in I N$. If $A x=\left(A_{n}(x)\right) \in Y$ for each $x=\left(x_{k}\right) \in X$, then $A$ defines a matrix mapping from $X$ into $Y$ and we denote it by $A: X \rightarrow Y .(X: Y)$ is the class of all matrices $A$ such that $A: X \rightarrow Y$. The domain $X_{A}$ is defined by

$$
X_{A}=\{x \in w: A x \in X\}
$$

which is a sequence space. If $A$ is triangle, then it can be easily shown that the sequence spaces $X_{A}$ and $X$ are linearly isomorphic, i.e., $X_{A} \cong X[1]$.

*Corresponding Author: mkarakas@beu.edu.tr 
A sequence space $X$ with a linear topology is called a $K$-space provided each of the maps $p_{n}: X \rightarrow C$ defined by $p_{n}(x)=x_{n}$ is continuous for all $n \in I N$, where $\mathrm{C}$ denotes the complex field and $I N=\{0,1,2, \ldots\}$. A $K$-space $X$ is called an $F K$-space provided $X$ is a complete linear metric space. An $F K$-space whose topology is normable is called a $B K$-space [2]. The spaces $l_{\infty}, c, c_{0}$ are $B K$-spaces with the sup-norm $\|x\|_{\infty}=\sup _{k}\left|x_{k}\right|$ and the space $l_{p}(1 \leq p<\infty)$ is $B K$-space with $\|x\|_{p}=\left(\sum_{k=0}^{\infty}\left|x_{k}\right|^{p}\right)^{1 / p}$.

The Fibonacci numbers are famous for possessing wonderful and amazing properties. Some of these properties are well-known. For instance, the sums and differences of Fibonacci numbers are Fibonacci numbers, and the ratios of Fibonacci numbers converge to the golden section, $\tau=\frac{1+\sqrt{5}}{2}$, which is important in Architecture, Nature and Art, physics [3].

The Fibonacci numbers $f_{n}$ are the terms of the sequence $0,1,1,2,3,5, \ldots$ where in each term is the sum of the preceding terms, beginning with the values $f_{0}=0$ and $f_{1}=1$. However, some fundamental properties of Fibonacci numbers are given as follows [4]:

$$
\begin{gathered}
\sum_{k=1}^{n} f_{k}=f_{n+2}-1 ; n \geq 1 \\
\sum_{k=1}^{n} f_{k}^{2}=f_{n} f_{n+1} \\
\left\{f_{k}\right\}_{k=1}^{\infty} \text { converges }
\end{gathered}
$$

In the present study, we define the matrix $F=\left(f_{n k}\right)_{n, k=1}^{\infty}$ using Fibonacci numbers $f_{n}$ and establish the sequence spaces $l_{p}(F), l_{\infty}(F), c(F)$ and $c_{0}(F)$ where $1 \leq p<\infty$. These spaces were also studied by different matrix in [5].

\section{Main Results}

Now, we state the well known Toeplitz theorem which gives the necessary and sufficient conditions for regularity of a matrix.

Theorem 2.1 [6, Lemma 2.1]. A matrix $A=\left(a_{n k}\right)_{n, k=1}^{\infty}$ is regular if and only if the following three conditions hold:

i. There exists $M>0$ such that for every $n=1,2,3, \ldots$ the inequality $\sum_{k=1}^{\infty}\left|a_{n k}\right| \leq M$ holds;

ii. $\quad \lim _{n \rightarrow \infty} a_{n k}=0$ for every $k=1,2, \ldots$;

iii. $\quad \lim _{n \rightarrow \infty} \sum_{k=1}^{\infty} a_{n k}=1$.

In consideration of the above information, we define the Fibonacci matrix $F=\left(f_{n k}\right)_{n, k=1}^{\infty}$ as follows: 


$$
\begin{aligned}
f_{n k} & =\left\{\begin{array}{l}
\frac{f_{2 k}}{f_{2 n+1}-1}, 1 \leq k \leq n \\
0, \text { otherwise }
\end{array},\right. \text { that is, } \\
F & =\left[\begin{array}{ccccccc}
1 & 0 & 0 & 0 & 0 & 0 & \ldots \\
\frac{1}{4} & \frac{3}{4} & 0 & 0 & 0 & 0 & \ldots \\
\frac{1}{12} & \frac{3}{12} & \frac{8}{12} & 0 & 0 & 0 & \ldots \\
\frac{1}{33} & \frac{3}{33} & \frac{8}{33} & \frac{21}{33} & 0 & 0 & \ldots \\
\vdots & \vdots & \vdots & \vdots & \vdots & \vdots & \ddots
\end{array}\right] .
\end{aligned}
$$

In connection with $f_{n n} \neq 0$ and $f_{n k}=0$ for $k>n$, the above matrix $F$ is triangle and also it can be easily seen by the Toeplitz theorem that the method $F$ is regular.

Hereby, we introduce the following Fibonacci sequence space where the sequence

$$
y=\left(y_{k}\right)=F_{k}(x)=\frac{1}{f_{2 k+1}-1} \sum_{i=1}^{k} f_{2 i} x_{i}
$$

is the $F$-transform of a sequence $x=\left(x_{k}\right)$ for all $k \in I N^{0}$ :

$$
X(F)=\left\{x \in w: F x=y=\left(y_{k}\right) \in X\right\} .
$$

Here and in the sequel, $X$ denotes any of the sequence spaces $l_{\infty}, c, c_{0}$ and $l_{p}(1 \leq p<\infty)$. We can redefine the space $X(F)$ with the notation (1.1) as follows:

$$
X(F)=X_{F} \text {. }
$$

Theorem 2.2. The space $X(F)$ is a $B K$ space with the norm

$$
\|x\|_{X(F)}=\|F x\|_{X}=\|y\|_{X}=\sup _{k}\left|y_{k}\right| \text { for } X \in\left\{l_{\infty}, c, c_{0}\right\}
$$

and also

$$
\|x\|_{X(F)}=\|F x\|_{X}=\|y\|_{X}=\left(\sum_{k=1}^{\infty}\left|y_{k}\right|^{p}\right)^{1 / p} \text { for } X=l_{p}(1 \leq p<\infty) .
$$

Proof: Since the matrix $F$ is triangle, (2.2) and Theorem 4.3.12 of Wilansky [7] gives the fact that the space $X(F)$ is $B K$-space with the above norms.

Theorem 2.3. The Fibonacci sequence space $X(F)$ is isometrically isomorphic to space $X$.

Proof: We should show the existence of an isometric isomorphism between the spaces $X(F)$ and $X$. Let us take in consideration the transformation $P$ defined from $X(F)$ to $X$ by $P: X(F) \rightarrow X, x \rightarrow P x=y, y=\left(y_{k}\right)=F_{k}(x)=\frac{1}{f_{2 k+1}-1} \sum_{i=1}^{k} f_{2 i} x_{i}$. In that case, for every $x \in X(F)$ we have $P x=y=F(x) \in X$. In addition, it is clear that $P$ is linear. Then, it can be easily seen that $P x=0 \Rightarrow x=0$ and so $P$ is injective.

Besides, let us define the sequence $x=\left(x_{k}\right)$ as follows: 


$$
x_{k}=\frac{f_{2 k+1}-1}{f_{2 k}} y_{k}-\frac{f_{2 k-1}-1}{f_{2 k}} y_{k-1} ; k \in I N^{0}, y=\left(y_{k}\right) \in X .
$$

Then, for every $k \in I N^{0}$ the following equality is obtained from (2.1) and (2.5):

$$
F_{k}(x)=\frac{1}{f_{2 k+1}-1} \sum_{i=1}^{k} f_{2 i} x_{i}=\frac{1}{f_{2 k+1}-1} \sum_{i=1}^{k}\left[\left(f_{2 i+1}-1\right) y_{i}-\left(f_{2 i-1}-1\right) y_{i-1}\right]=y_{k} .
$$

It means that $F x=y$ and thus we get that $F x \in X$ as $y \in X$. By this way, we conclude that $x \in X(F)$ and $P x=y$. As a consequence, $P$ is surjective. Additionally, it follows from (2.3) and (2.4) that $P$ is norm preserving, that is,

$$
\|P x\|_{X}=\|y\|_{X}=\|F(x)\|_{X}=\|x\|_{X(F)}
$$

for any $x \in X(F)$. Hence $P$ is isometry. Accordingly, the spaces $X(F)$ and $X$ are isometrically isomorphic, that is, $X(F) \cong X$.

Lemma 2.4. Let $\left\{f_{k}\right\}_{k=1}^{\infty}$ be Fibonacci number sequence. If the sequence $\left(\frac{1}{f_{2 k+1}-1}\right)$ is in $l_{1}$, then $\sup _{i}\left(f_{2 i} \sum_{k=i}^{\infty} \frac{1}{f_{2 k+1}-1}\right)<\infty$.

Proof: It can be easily seen that the sequence $\left(\frac{1}{f_{2 k+1}-1}\right)$ is in $l_{1}$. So, the result follows from Lemma 4.11 of Mursaleen and Noman [8].

Theorem 2.5. For $X=c_{0}, c, l_{\infty}$ the inclusion $c_{0}(F) \subset c(F) \subset l_{\infty}(F)$ strictly holds.

Proof: It is clear that the inclusion $c_{0}(F) \subset c(F) \subset l_{\infty}(F)$ holds. Consider the sequence $x=\left(x_{i}\right)$ defined by $x_{i}=1$ for all $i \in I N^{0}$. Then we have for every $k \in I N^{0}, F_{k}(x)=\frac{1}{f_{2 k+1}-1} \sum_{i=1}^{k} f_{2 i}=1$. Hence, it is obvious that $F x \in c$ but it is not in $c_{0}$. So the sequence $x$ is in $c(F)$ but $x \notin c_{0}(F)$. Consequently, the inclusion $c_{0}(F) \subset c(F)$ is strict. Now, let us consider the sequence $x_{i}=\frac{(-1)^{i}\left(f_{2 i+1}+f_{2 i-1}-1\right)}{f_{2 i}}$ for all $i \in I N^{0}$. By this way, we have $F_{k}(x)=\frac{1}{f_{2 k+1}-1} \sum_{i=1}^{k} f_{2 i} x_{i}=(-1)^{k}$ for every $k \in I N^{0}$. This shows that $F x \in l_{\infty}$ but not in $c$. Thus, it is clear that $x \in l_{\infty}(F)$ but $x \notin c(F)$. Hereby, the inclusion $c(F) \subset l_{\infty}(F)$ is strict.

Theorem 2.6. The inclusion $X \subset X(F)$ holds.

Proof: Since the matrix $F$ is regular, the inclusion is obvious for $X=c_{0}, c$. If we take $x=\left(x_{i}\right) \in l_{\infty}$, then there is a constant $M>0$ such that $\left|x_{i}\right| \leq M$ for all $i \in I N^{0}$. Thus, we obtain the following inequality which gives that $F x \in l_{\infty}$ : 


$$
\left|F_{k}(x)\right| \leq \frac{1}{f_{2 k+1}-1} \sum_{i=1}^{k} f_{2 i}\left|x_{i}\right| \leq \frac{M}{f_{2 k+1}-1} \sum_{i=1}^{k} f_{2 i}=M .
$$

Hence, we conclude that $x=\left(x_{i}\right) \in l_{\infty} \Rightarrow x=\left(x_{i}\right) \in l_{\infty}(F)$. Now let us take $x=\left(x_{i}\right) \in l_{p}$, $1<p<\infty$. By using the Hölder's inequality, we have for every $k \in I N^{0}$ the following inequality:

$$
\left|F_{k}(x)\right|^{p} \leq\left[\sum_{i=1}^{k} \frac{f_{2 i}}{f_{2 k+1}-1}\left|x_{i}\right|\right]^{p} \leq\left[\sum_{i=1}^{k} \frac{f_{2 i}}{f_{2 k+1}-1}\left|x_{i}\right|\right]^{p}\left[\sum_{i=1}^{k} \frac{f_{2 i}}{f_{2 k+1}-1}\right]^{p-1}=\frac{1}{f_{2 k+1}-1} \sum_{i=1}^{k} f_{2 i}\left|x_{i}\right|^{p} .
$$

The inequality (2.6) gives the fact that

$$
\sum_{k=1}^{\infty}\left|F_{k}(x)\right|^{p} \leq \sum_{k=1}^{\infty} \frac{1}{f_{2 k+1}-1} \sum_{i=1}^{k} f_{2 i}\left|x_{i}\right|^{p}=\sum_{i=1}^{\infty}\left|x_{i}\right|^{p} f_{2 i} \sum_{k=i}^{\infty} \frac{1}{f_{2 k+1}-1} .
$$

For $\sup _{i}\left(f_{2 i} \sum_{k=i}^{\infty} \frac{1}{f_{2 k+1}-1}\right)<\infty$, it follows from lemma 2.4 that

$$
\|x\|_{l_{p}(F)}^{p} \leq M \sum_{k=1}^{\infty}\left|x_{i}\right|^{p}=M\|x\|_{l_{p}}^{p} .
$$

Hence, we have $x \in l_{p}(F)$ and so $l_{p} \subset l_{p}(F)$ for $1<p<\infty$. For $p=1$, it can be similarly shown that (2.7) holds. To prove that the converse of Theorem 2.6 holds, we'll use the matrix $\Lambda=\left(\lambda_{n k}\right)$ defined by $\lambda_{n k}=\left\{\begin{array}{c}\frac{\lambda_{k}-\lambda_{k-1}}{\lambda_{n}},(1 \leq k \leq n) \\ 0,(k>n)\end{array}\right.$ where $\lambda=\left(\lambda_{k}\right)_{k=0}^{\infty}$ is strictly increasing sequence of positive reals tending to infinity in [9]. In the special case $\lambda_{n}=f_{2 n+1}-1$, we have $\lambda_{k}-\lambda_{k-1}=f_{2 k}$ and so $F=\Lambda$ for every $k \in I N^{0}$. In these premises, we have that

$$
\lim _{n \rightarrow \infty} \frac{\lambda_{n+1}}{\lambda_{n}}=\lim _{n \rightarrow \infty} \frac{f_{2 n+3}-1}{f_{2 n+1}-1}=\lim _{n \rightarrow \infty}\left(1+\frac{f_{2 n+2}}{f_{2 n+1}-1}\right)=1+\lim _{n \rightarrow \infty} \frac{f_{2 n+2}}{f_{2 n+1}-1}>1 .
$$

Consequently, we obtain from [9, corollary 4.7] that $X(F) \subset X$ for $X=\left\{c_{0}, c, l_{p}\right\} \quad$ where $1 \leq p \leq \infty$.

Since the inclusions $X(F) \subset X$ and $X \subset X(F)$ hold, we can give the following result:

Corollary 2.7. $X=X(F)$.

\section{References}

1. Başar F, 2011. Summability Theory and Its Applications, Bentham e-Books, 410s. İstanbul.

2. Choudary B, Nanda S, 1989. Functional Analysis with Applications, John Wiley \& Sons Inc, New Delhi, India, 272-273.

3. Kalman D, Mena R, 2003. The Fibonacci Numbers: Exposed, Mathematics Magazine, 76 (3): 167-181.

4. Vajda S, 1989. Fibonacci and Lucas Numbers, and the Golden Section: Theory and Applications, Dover Publications Inc., 190 s. New York. 
5. Kara EE, Basarir M, Mursaleen M, 2012. Compact Operators on the Fibonacci Difference Sequence Spaces $\ell_{p}(\hat{F})$ and $\ell_{\infty}(\hat{F})$, 1st International Eurasian Conference on Mathematical Sciences and Applications, pp 45, September 3-7, Prishtine, Kosovo.

6. Kara EE, Basarir M, 2012. An Application of Fibonacci Numbers into Infinite Toeplitz Matrices, Caspian Journal of Mathematics Sciences, 1 (1): 1-6.

7. Wilansky A, 1984. Summability Through Functional Analysis, Elsevier Science Publishers B.V., 309 s. Amsterdam.

8. Mursaleen M, Noman AK, 2011. On Some New Sequence Spaces of Non-absolute Type Related to the Spaces $l_{p}$ and $l_{\infty}$ I, Filomat, 25 (2): 33-51.

9. Mursaleen M, Noman AK, 2010. On the Space of $\lambda$-Convergent and Bounded Sequences, Thai Journal of Mathematics, 8 (2): 311-329.

Geliş Tarihi: 21/10/2015

Kabul Tarihi: 09/12/2015 